\title{
Development of a Mobile-app based free field click test against conventional free field voice test: A randomized controlled trial
}

\author{
THILAGA RAJENDRAN ${ }^{1}$, Julius Goh Liang Chye $^{1}$, and Jothi Shanmuganathan ${ }^{1}$ \\ ${ }^{1}$ Hospital Sultanah Aminah
}

September 21, 2020

\begin{abstract}
Objective:Hearing loss is a prevalent disease with free field voice test (FFVT) being a common hearing test performed.FFVT has its own disadvantage as a screening tool as there is no standardization of speech among examiners. We aim to invent mobile-app based hearing screening using Flutter-Dart programming language that mimics findings of FFVT.The app is termed as Free field click test (FFCT). Our aim is to compare sensitivity\&specificity of FFCT and FFVT with Pure Tone audiogram (PTA). Methods:This is an open-labelled randomized controlled trial. This study was conducted at the (removed for blind peer review) from December-March 2020. 50 patients from 18-80 years old with complaint of hearing loss were recruited.These patients were randomly assigned into two groups; FFVT\&FFCT which were compared with PTA.Categorical data were analyzed with Chisquare test,non-categorical data were calculated with Student t-test for statistical significance. Results:Results were calculated based on degree of hearing loss against PTA.FFCT's rate of detection of hearing loss was significant for all degrees of hearing loss with p-values of $<0.05$. FFVT's detection rate was only significant to normal hearing, moderate \& severe hearing loss. FFCT had higher sensitivity of $94.1 \%$ for normal hearing compared to $52 \%$ for FFVT.FFVT had higher specificity but with a marginal difference only.A higher rate of accuracy (94\%) and strength of the correlation (0.97) were obtained for FFCT than FFVT. Conclusion:FFCT is a more sensitive\&accurate tool than FFVT in detecting hearing thresholds. It proves to be an ideal tool to screen hearing loss and is convenient in times of social distancing during COVID-19 pandemic now.
\end{abstract}

Key points

1.To overcome the disadvantages of the current hearing screening methods

2. To eliminate the disadvantages of the conventional FFVT

3.To invent a digital option to screen hearing loss

3. To test the accuracy and sensitivity of the mobile-app and FFVT compared to PTA

4. To have more accessibility to screen hearing loss nationwide

Level of evidence : Level 2

Keywords : mobile apps; smartphone; audiometry; hearing loss; hearing tests

\section{INTRODUCTION}

Hearing loss is a prevalent disease in the population. This disorder does not only affect aging population but, also younger adults and children. In children, this condition can implicate their normal speech and language development. According to World Health Organization, about 466 million people have disabling hearing loss globally, which is about $5 \%$ of the world's overall population. Among these vast numbers, 34 million are children. It is predicted that the number will escalate to over 900 million people by 2050, in which one in ten people will have the hearing loss disability. 
There are various tests available to detect hearing loss in the clinical settings, which can be either objective or subjective tests.. Tuning fork, pure tone audiometry (PTA) and Barany box are the examples. The commonest subjective test used is PTA. Hearing loss is tested with audiometry by average of hearing thresholds at frequencies of $0.5 \mathrm{kHz}, 1 \mathrm{kHz}, 2 \mathrm{kHz}$, and $4 \mathrm{kHz}$ while hearing loss of $35 \mathrm{~dB}$ and more are viewed as disabling hearing losses. ${ }^{2}$ However, PTA is not a practical solution for hearing screening in a large population as it requires trained audiologists to conduct the test. ${ }^{3}$ Moreover, not every healthcare facility own an audiology service as a soundproof room with an audiometry are required. ${ }^{4,5}$

Free Field Voice Test (FFVT) or also known as whispered voice test is another screening tool to detect hearing loss. This test can be used for both children and adults as it uses a combination of numbers and letters. In children, bi-syllabic words like cowboy and football are often used. ${ }^{6}$ A study on FFVT reported that the test had $90 \%$ test sensitivity in detecting hearing thresholds above $30 \mathrm{~dB}$ HL, making it a suitable hearing screening tool. ${ }^{7}$ General practitioners are often recommended to use FFVT to screen hearing among the elderly. Clinical guidelines are also formulated by various national health board to make hearing impairment as part of the health screening. ${ }^{8}$ However, FFVT have been criticized as there is no standardization of sound levels of speech among the examiners. There is also a significant difference in the sound levels of the examiners tested in different occasions, especially during a whisper. ${ }^{6}$

With usage of mobile phones being an integral part of our daily lives, many health-related apps are easily accessible now. Hearing assessments can also be conducted via mobile phones which are portable, cost-effective and accessible especially in resource-limited settings. ${ }^{5}$ There is a growing interest in establishing more of such digital services such as smartphone-enabled otoscope and noise level detectors. ${ }^{9}$ In a recent systematic review published, the validation of smartphone apps in supporting and controlling tinnitus symptoms was reported. One of the relief therapies analyzed is the mobile phone-based Cognitive Behavioural Therapy (CBT) which proved to be beneficial to patients with tinnitus, which frequently accompanies hearing loss. ${ }^{10}$

Our aim is to create a cost-effective, digital tool to screen hearing. Thus, we have designed an app using Flutter-Dart language, which is a programming tool used to create cross- platform mobile applications. Mobile apps can be used to mimic the findings of FFVT and is dubbed by the authors as the free field click test (FFCT). It is named as such as it employs broad-band clicks to elicit a clinical response. There is very little information in the literature about the validity of digital hand-held devices on the detection of hearing loss. This study will demonstrate on how a low-cost software can be developed and used to detect hearing loss in the general population in the future.

\section{MATERIALS \& METHODS}

\section{Study Population}

This study was conducted at a sound-proof room in ORL clinic in/removed for blind peer review] from December till March 2020. A total of 50 patients from the age of 18 until 80 years old with primary complaint of hearing loss were recruited. These patients were then randomly assigned into two groups; FFVT and FFCT which were then compared with the gold standard of PTA. Patients whom were less than 18 and more than 80 years old with hearing loss and pregnant ladies were excluded from this study. Patients with less than one year duration of post-traumatic and post-operative hearing loss were also excluded.

Study design

\section{Procedures}

This is an open-labelled randomized controlled trial involving two groups which are FFVT vs FFCT (digital hand-held device). The app is designed by our team using the Flutter-Dart programming tool and the speaker is produced via hand-held devices of Apple and Android brands. Two types of sound were created which represent the individual sounds from FFVT, which are soft sounds (whisper) ranging from 30-40dB and loud sounds (conversation voice) ranging from $60-70 \mathrm{~dB}$. These click sounds range from $500-3000 \mathrm{~Hz}$, which corresponds to the standard pure-tone average values. Calibration of sounds produced from the mobile app 
was done using an audiometer at distances of $60 \mathrm{~cm}$ and $15 \mathrm{~cm}$. The tests were all done in a soundproof room.

The examiner initially stands at a distance of $60 \mathrm{~cm}$ (full arm's length) behind a seated patient to prevent lip reading. The patient's eyes were shielded and tragal rubbing of the non-test ear was performed. For FFVT, a sequence of spondee/bisyllable words was whispered and the patient repeats it. If the patient fails to hear the whispered words at $60 \mathrm{~cm}$, the examiner then moves closer to $15 \mathrm{~cm}$ (half arm's length) and whispers the spondee words again. If no response is obtained, conversational voice at $60 \mathrm{~cm}$ and lastly conversational voice at $15 \mathrm{~cm}$ distances are used if the patient is still unable to hear.${ }^{11}$ For each distance, the words were repeated thrice and a different set of Spondee words was used. The patient's hearing threshold was the voice and distance level at which more than $50 \%$ of words were repeated correctly. [Figure 1]

The same technique was conducted for FFCT but, instead of whispering, we used the soft and loud sounds from the mobile app [Figure $1 \& 2$ ]. The distances used were also similar to FFVT, with $60 \mathrm{~cm}$ initially and then moving closer to $15 \mathrm{~cm}$. The sounds were produced 3 times, with 2 out of 3 answers were considered as the patient's hearing threshold.

Sample size calculation

The sample size was determined by using a power study via a web-based sample size calculator(https://clincalc.com/stats/samplesize.aspx). The sample size calculation was based on the reference data from the National ORL registry of hearing-related diseases in $2011 .{ }^{12}$ We estimated that we would expect $86 \%$ sensitivity and $90 \%$ specificity while performing the FFVT. Hence, we have considered a $90 \%$ power and $5 \%$ marginal error, which leads to a minimum sample size of 25 patients/50 ears per group. Randomization was done using a web-based randomizer:http://www.graphpad.com/quickcalcs/randomize1@7th December 2019@1500H . The total number of patients was separated into 2 groups and each group contained 25 samples (50 ears). The numbers were randomized and printed according to the web-based randomizer at the aforementioned date and time. Generated numbers are then saved for further reference.

Statistical Analysis Plan

Based on the studies reported by Malaysian National Otorhinolaryngology of Hearing-related Diseases, ${ }^{12}$ the prevalence of hearing impairment was reported to be approximately $50 \%$, and thus, we estimated that we would expect an $86 \%$ sensitivity and $90 \%$ specificity while performing a free field voice test. ${ }^{8,13}$ Descriptive data were expressed as mean \pm standard deviation (SD) unless otherwise stated. Categorical data were analyzed using Chi-square test while, non-categorical data were calculated using Student t-test.A value of $\mathrm{P}$ $<0.05$ was considered statistically significant. The data collected were analyzed using an intention-to-treat basis.

\section{RESULTS}

\subsubsection{Demographic details}

The minimum age of participants was 18 years old while, the maximum age was 80 years old. $52 \%$ of the participants were male and $62 \%$ came with the complaint of unilateral hearing loss. $40 \%$ of our participants had no additional symptoms besides the main complaint of hearing loss. $33 \%$ of participants had tinnitus, $11 \%$ had associated vertigo or giddiness while $5 \%$ had otorrhea. Other additional symptoms like rhinitis, otalgia, headache and facial weakness were reported to be less than $5 \% .45 \%$ of participants had normal hearing when PTA was performed. $28 \%$ of participants had mild hearing loss, $17 \%$ had moderate hearing loss and severe hearing loss had $9 \%$ of participants. Only $1 \%$ had profound hearing loss. FFCT took an average of 1 minute to be completed for $60 \%$ participants. FFVT took an average of 2-3 minutes.

\subsubsection{Analysis of hearing loss of FFVT \& FFCT}

The distribution of degree of hearing loss for FFVT as compared to PTA showed the p-values in correspondence to each participant were $<0.05$ for all degrees of hearing loss except for mild hearing loss. [Table 1] For FFCT, the p-values in correspondence to each participant were $<0.05$ for all degrees of hearing loss. But, 
the p-value for profound hearing loss was insignificant as there was insufficient sample to be analysed. Both FFCT and FFVT thresholds correlate and are dependent on the audiogram values for all degrees of hearing loss, except for mild hearing loss in FFVT.

\subsubsection{Comparison of FFVT \& FFCT}

FFCT's rate of detection was significant for all degrees of hearing loss. This is in comparison to FFVT where the detection rate was only significant for normal hearing, moderate and severe hearing loss. FFCT had a higher sensitivity of $94.1 \%$ for normal hearing than $52 \%$ for FFVT. [Figure 4] However, with a marginal difference, FFVT was found to have a higher specificity than the click test, which was $96 \%$ compared to $93.9 \%$. Yet, FFCT proved to have higher specificity in detecting other levels of hearing loss. [Figure 5] FFCT had performed with a higher rate of accuracy (94\%) than FFVT (74.9\%). A higher strength of the correlation, 0.97 was tabulated in conducting FFCT than FFVT(0.96).

\subsection{DISCUSSION}

The outcomes from this study are evident to support the use of the mobile-app based FFCT to screen hearing loss. This digital method of hearing screening is the pioneer study carried out in a clinical practice in Malaysia. The limitations of voice test and audiogram can thus be eliminated. A developmental study conducted on smartphone-connected hearing devices proved that the usage was higher than conventional hearing aids. ${ }^{14}$

FFVT on the other hand can be performed using words, numbers or phrases to suit the age of patients. A study conducted using certain higher frequency speech stimuli proved that FFVT is an acceptable hearing screening in the geriatric population. Phonetically-balanced words or phrases were included to increase the reproducibility of the testas loss of hearing at higher frequencies is a feature of presbycusis. ${ }^{15} \mathrm{~A}$ systematic review was conducted to determine the accuracy of whispered voice test. Though the sensitivity in four of the adult studies was 90-100\%, there was no standardization of loudness of the whisper among the examiners and no proper spoken sequence of words. The distance between the examiner and patient also varied. ${ }^{8}$ Thus, these factors affect the accuracy and reproducibility of the test. The effectiveness of FFVT using numbers and phonetically balanced monosyllabic (PB) words were analysed in a comparative study. It is revealed that numbers or easily recognizable words were effortlessly identified and repeated by patients than PB words. But, the real relationship of hearing threshold and hearing distance were not very accurate. It was revealed that $42 \%$ of patients who passed FFVT using numbers were found to have hearing loss when tested using PB words later. This was applicable mainly in patients with high-frequency hearing loss, such as presbycusis and acoustic trauma. ${ }^{16}$

Hearing screening with PTA is the gold standard test. PTA is performed by trained audiologists with an instrument that creates a standard range of test stimuli set at premeasured loudness of sound using decibels. The American Speech-Language-Hearing Association and the American Academy of Audiology had given recommendations that define screening at $20 \mathrm{~dB}$ at frequencies of 1000,2000 and $4000 \mathrm{~Hz}$. Thus, PTA was the gold standard test in our study that was compared with FFVT and FFCT. ${ }^{17}$ PTA serves as a significant apparatus to determine the type and degree of hearing loss involved in the diagnosis of various otologicalrelated diseases. PTA plays key role in helping clinicians with surgical decisions and to monitor treatment progress. However, the audiogram is a relatively old method to screen hearing, and it has only advanced minimally over the past decades. ${ }^{18}$ The limited audiology facilities and expertise are its drawbacks too. PTA is usually not available in the general practice or district hospitals. A long waiting time is almost always experienced by patients to have their hearing screened by audiologists, thus, causing more delays in treatment and rehabilitation services.

There are various hearing-related mobile-based apps available online. However, there is no hearing app found so far which can be incorporated into our daily otolaryngology practice. Most of the apps are not validated against PTA and thus rendering them not suitable to be used as screening tools. During the World Hearing Day in 2019, the World Health Organization had developed a mobile and web-based software for hearing screening. The software is known as 'hear WHO app'. This app was based on validated digits-in- 
noise technology. It is useful in monitoring hearing of people with noise exposure at workplace and avid earphone users. But, as the app is language-dependent, it demands further validation and conversion into different languages prior to its worldwide usage. ${ }^{19} \mathrm{~A}$ review was performed by Bright et.al. in 2016 on smartphone-based hearing apps. About 30 apps were chosen from Google Play and Apple App Store. These apps were validated against PTA but, their accuracies varied. ${ }^{20}$ This review concluded that more detailed, independent validation studies are required to further test the accuracy of these apps for clinical practice. Another review was conducted among army servicemen in India to compare the sensitivity of FFVT with a free iOS application to detect hearing loss. It was reported that the hearing check app was more sensitive than the conventional voice test $(98 \%$ and $73 \%) .{ }^{21}$

We report that in our study FFCT has a more standard way of examination with constant broadband sounds produced in correspondence to pure tone average. This differs from FFVT as there was a lack in the standardization of speech sound levels of the examiners. FFCT is user friendly as it only requires a mobile phone/tablet with an iOS/Android operating system to access the app. It is much easier for patients to acknowledge the sounds produced instead of repeating the spondee words used in FFVT. This is especially useful in patients with presbycusis and with language problems. FFCT requires minimal practice before one can start to operate it. There was no cost involved as our team incorporated basic programming skills to develop the app. FFCT was able to be completed faster than FFVT with the average time of 1 minute. FFCT was shown to be more sensitive than FFVT while, FFVT has a higher specificity for normal hearing levels. [Table 2] FFCT has numerous potential uses as it can provide a rapid test to predict hearing loss and can be easily accessible by medical personnel as the programming language is open-sourced. It is a suitable tool to screen hearing when audiograms are not available, especially during night shifts and in district hospitals. FFCT is a convenient way to monitor treatment response such as in sudden-onset sensorineural hearing loss. It serves as a teaching tool and as a beginner's teaching module for doctors interested in learning programming subject whereby more health-related mobile apps or medical devices can be developed.

Given the COVID-19 pandemic presently, hearing screening can be rather challenging to be carried out. Avoiding close contacts between hospital staffs and patients are the utmost priority to be adhered upon and hence, FFCT can be very useful in times of crises as such. With social distancing we can still carry out hearing screening safely while minimizing community transmission of this deadly virus among healthcare workers. ${ }^{22}$ Hearing screening via mobile-app has an added advantage of being able to perform faster especially in crowded hospitals. FFCT is user-friendly for patients whom prefer to maintain appropriate physical contact and distance from the examiners.

\subsection{LIMITATIONS OF THE STUDY}

Both FFVT and FFCT were conducted in an enclosed, soundproof room. This room might not have reflected the environmental noise which is present during the routine FFVT. However, this limitation is in accordance with the RCT study design. No environmental noise was involved which could have altered the intensity of sound present in the soundproof room. The examination distance varied slightly from each examiner to another. This study was compared against PTA, which itself is one of the subjective assessment of hearing. Objective hearing test that can be performed is Auditory Brainstem Response (ABR). Only a single programming language was used to create the mobile app with which the features of mobile app was not able to be compared upon.

\subsection{CONCLUSION}

This RCT paper proposes that the mobile-app based FFCT designed using Flutter-Dart programming language stands to be an ideal tool to screen hearing loss in the general population. FFCT is a more sensitive \& accurate tool than conventional FFVT in detecting hearing thresholds. This cost-effective app is a sign ofdigital modernization and will hopefully be the first clinical app of many apps to be developed by future clinical programmers.

\section{ABBREVIATIONS}


HL - hearing loss

$\mathrm{RCT}$ - randomized controlled trial

PTA - pure tone audiometry

FFVT - free field voice test

FFCT - free field click test

COVID-19 - Coronavirus disease 2019

FINANCIAL DISCLOSURE \& CONFLICT OF INTEREST

This research did not receive any specific grant from funding agencies in the public, commercial, or not-forprofit sectors.

The authors have no financial disclosures or conflicts of interest to declare.

ETHICS OF THE STUDY

The researchers obtained the approval and study registration from the Medical Research and Ethics Committee (MREC) via National Medical Research Register (blinded for review). The study is also registered in the United States National Library of Medicine, a clinical trial registry. (ClinicalTrials.gov Identifier: blinded for review ). The study has been listed as a World Health Organization (WHO)-approved study.

\section{REFERENCES}

Single-sided deafness recognised as a disability in Malaysia.

https://www.thestar.com.my/lifestyle/health/2019/12/11/recognised-as-a-disability. Accessed January 10, 2020.

1. Wilson BS, Tucci DL, Merson MH, O'Donoghue GM. Global hearing health care: new findings and perspectives. Lancet. 2017;390(10111):2503-2515.

2. Bexelius C, Honeth L, Ekman A, et al. Evaluation of an internet-based hearing test-comparison with established methods for detection of hearing loss. J Med Internet Res. 2008;10(4):e32.

3. Derin S, Cam OH, Beydilli H, Acar E, Elicora SS, Sahan M. Initial assessment of hearing loss using a mobile application for audiological evaluation. J Laryngol Otol . 2016;130(3):248-251.

4. Barczik J, Serpanos YC. Accuracy of Smartphone Self-Hearing Test Applications Across Frequencies and Earphone Styles in Adults. Am J Audiol . 2018;27(4):570-580.

5. Open access guide to audiology \& hearing aids for otolaryngologists. http://www.entdev.uct.ac.za. Accessed January 25, 2020.

6. Dempster JH, Mackenzie K. Clinical role of free-field voice tests in children. ClinOtolaryngol Allied Sci. 1992;17(1):54-56.

7. Pirozzo S, Papinczak T, Glasziou P. Whispered voice test for screening for hearing impairment in adults and children: systematic review. BMJ. 2003;327(7421):967.

8. Renda L, Selcuk OT, Eyigor H, Osma U, Yılmaz MD. Smartphone Based Audiometric Test for Confirming the Level of Hearing; Is It Useable in Underserved Areas?. J IntAdv Otol . 2016;12(1):61-66.

9. Mehdi M, Riha C, Neff P, et al. Smartphone Apps in the Context of Tinnitus: Systematic Review. Sensors (Basel). 2020;20(6):1725.

10. Free field hearing test. https://entsho.com/ear-examination/. Accessed April 12, 2020.

11. The annual report of national ORL registry. (2011). Hearing and otology related disease/cochlear implant (vol.1). http://www.moh.gov.my/index.php/pages/view/1346. Accessed January 11, 2020.

12. McShefferty D, Whitmer WM, Swan IR, Akeroyd MA. The effect of experience on the sensitivity and specificity of the whispered voice test: a diagnostic accuracy study. BMJ Open. 2013;3(4):e002394. 
13. Maidment DW, Ferguson M. An Application of the Medical Research Council's Guidelines for Evaluating Complex Interventions: A Usability Study Assessing Smartphone-Connected Listening Devices in Adults With Hearing Loss. Am J Audiol . 2018;27(3S):474-481.

14. Labanca L, Guimarães FS, Costa-Guarisco LP, Couto EAB, Gonçalves DU. Screening of hearing in elderly people: assessment of accuracy and reproducibility of the whispered voice test. Triagemauditivaemidosos: avaliação da acurácia e reprodutibilidade do teste do sussurro. CienSaude Colet. 2017;22(11):3589-3598

15. Flottorp G. A comparison of the effectiveness of numbers versus PB words in voice tests of hearing. ActaOtolaryngol. 1959;50(1):13-25.

16. Chu YC, Cheng YF, Lai YH, Tsao Y, Tu TY, Young ST, Chen TS, Chung YF, Lai F, Liao WH. A Mobile Phone-Based Approach for Hearing Screening of School-Age Children: Cross-Sectional Validation Study JMIR MhealthUhealth 2019;7(4):e12033

17. Musiek FE, Shinn J, Chermak GD, Bamiou DE. Perspectives on the Pure-Tone Audiogram. J Am AcadAudiol. 2017;28(7):655-671. doi:10.3766/jaaa.16061

18. Swanepoel W, De Sousa KC, Smits C, Moore DR. Mobile applications to detect hearing impairment: opportunities and challenges. Bull World Health Organ. 2019;97(10):717-718.

19. Bright T, Pallawela D. Validated Smartphone-Based Apps for Ear and Hearing Assessments: A Review. JMIR Rehabil Assist Technol. 2016;3(2):e13.

20. Swami H, Bhargava A, Sabarigirish K, Arvind BM. A comparative study of smartphone based app with free field hearing for possible use as a screening test.

Int J Otorhinolaryngol Head Neck Surg. 2017;3:710-4.

Arora VM, Marius Chivu, MD, Andrew Schram, MD, MBA, Meltzer D, Implementing Physical Distancing in the Hospital: A Key Strategy to Prevent Nosocomial Transmission of COVID-19. J. Hosp. Med 2020;5;290291.

Figure/Table legends

Figure 1 Illustrations depicting the method of examinations A) FFVT at $60 \mathrm{~cm}, \mathrm{~B}) \mathrm{FFVT}$ at $15 \mathrm{~cm}, \mathrm{C}) \mathrm{FFCT}$ at $60 \mathrm{~cm}, \mathrm{D}) \mathrm{FFCT}$ at $15 \mathrm{~cm}$

Figure 2 Screenshot of FFCT mobile app with soft sound on top and loud sound below

Figure 3 Consort diagram showing the flow chart of the RCT study

Table 1 Distributions of hearing loss with FFVT \& FFCT compared with Audiogram

Figure 4 Sensitivity of FFVT vs FFCT for normal, mild, moderate \& severe hearing loss

Figure 5 Specificity of FFVT vs FFCT for normal, mild, moderate \& severe hearing loss

Table 2 Overall comparisons between FFVT vs FFCT

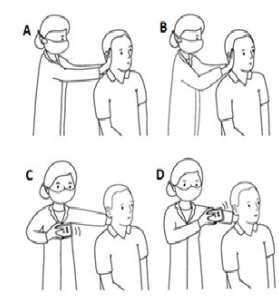



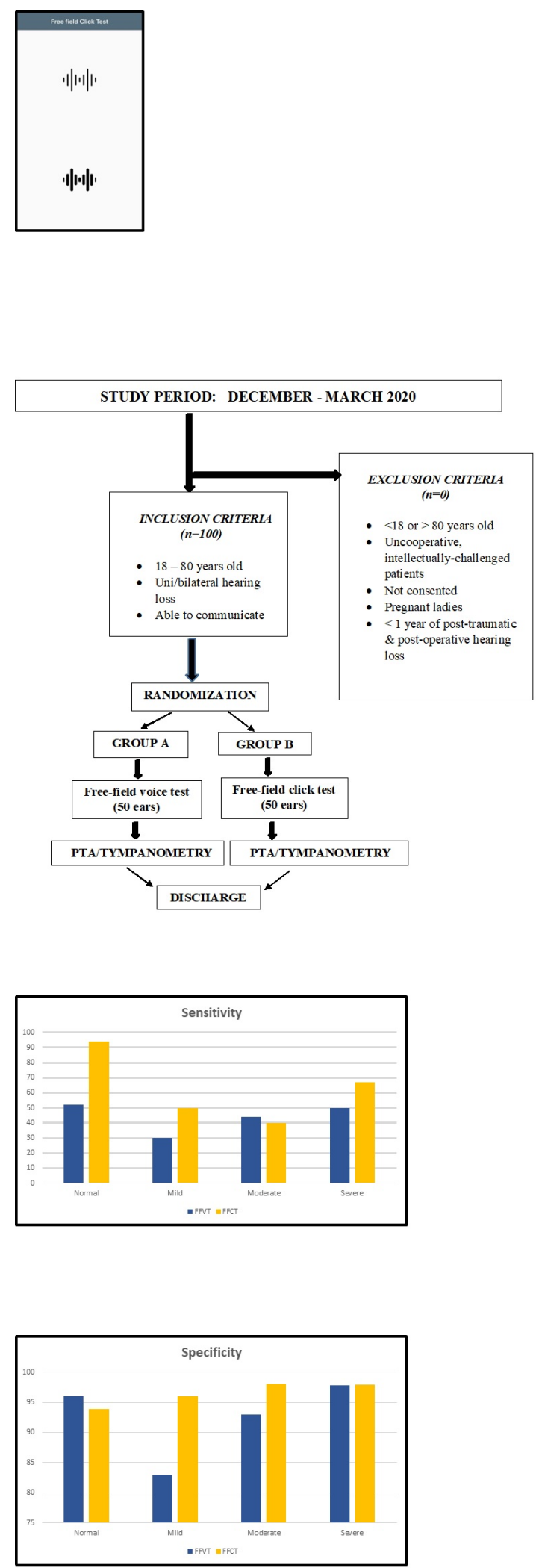


\section{Hosted file}

Table 1.docx available at https://authorea.com/users/359957/articles/481707-development-ofa-mobile-app-based-free-field-click-test-against-conventional-free-field-voice-test-arandomized-controlled-trial

\section{Hosted file}

Table 2.docx available at https://authorea.com/users/359957/articles/481707-development-ofa-mobile-app-based-free-field-click-test-against-conventional-free-field-voice-test-arandomized-controlled-trial 